Multistrip synthetic single-crystal-diamond photodiode based on a p-type/intrinsic/Schottky metal transverse configuration

This article has been downloaded from IOPscience. Please scroll down to see the full text article.

2011 EPL 9428004

(http://iopscience.iop.org/0295-5075/94/2/28004)

View the table of contents for this issue, or go to the journal homepage for more

Download details:

IP Address: 160.80 .88 .68

The article was downloaded on 19/03/2012 at 15:34

Please note that terms and conditions apply. 


\title{
Multistrip synthetic single-crystal-diamond photodiode based on a p-type/intrinsic/Schottky metal transverse configuration
}

\author{
I. Ciancaglioni ${ }^{1}$, Marco Marinelli $^{1}$, E. Milani ${ }^{1}$, G. Prestopino ${ }^{1}$ (a), C. Verona ${ }^{1}$, G. Verona-Rinati $^{1}$,

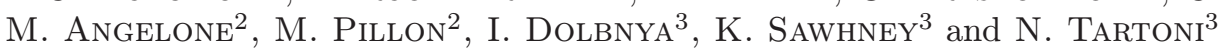 \\ ${ }^{1}$ INFN-Dipartimento di Ingegneria Meccanica, Università di Roma "Tor Vergata" - Via del Politecnico 1, \\ I-00133 Roma, Italy, EU \\ ${ }^{2}$ Associazione EURATOM-ENEA sulla Fusione - Via E. Fermi 27, I-00044 Frascati (Roma), Italy, EU \\ ${ }^{3}$ Diamond Light Source, Harwell Science and Innovation Campus - Chilton-Didcot, OX11 ODE Oxfordshire, UK, EU
}

received 24 January 2011; accepted in final form 15 March 2011

published online 18 April 2011

PACS 81.05.ug - Diamond

PACS 29.20.dk - Synchrotrons

PACS 89.20.Bb - Industrial and technological research and development

\begin{abstract}
A synthetic multistrip single-crystal-diamond detector based on a $p$-type/intrinsic diamond/Schottky metal transverse configuration, operating at zero-bias voltage, was developed. The device was characterized at the Diamond Light Source synchrotron in Harwell (UK) under monochromatic high-flux X-ray beams from 6 to $20 \mathrm{keV}$ and a micro-focused $10 \mathrm{keV}$ beam with a spot size of $\sim 3 \mu \mathrm{m}$. No significant pixel-to-pixel variation of both spectral responsivity and time response, high spatial resolution and good signal uniformity along each strip were found, suggesting the tested device structure as a promising sensor for X-ray and UV radiation imaging.
\end{abstract}

Detection of soft X-rays (SXRs) and deep UV (DUV) radiation has long been increasing the development of compact, solid-state, visible blind detectors based on wide-band-gap materials. In particular, advanced industrial and scientific research applications, such as new experimental techniques in synchrotron beamlines and astronomical observations, novel photolithographic processes and radiation diagnostic in magnetic-confinement nuclear-fusion reactors, demand high-performance radiation-hard devices able to effectively work even in extreme operating conditions, where high temperatures or high ionizing-particle fluxes are present. Moreover, high-spatial-resolution detectors for radiation imaging and in situ beam monitoring are also needed to optimize characterization methods.

Due to its exceptional chemical, physical and electronic properties $[1,2]$ diamond has been recognized as an ideal material for responding to these challenges. A great effort has been devoted by several research groups to improve both material quality and device design. In the last few years the availability of low-cost high-pressure high-temperature (HPHT) single-crystal-diamond (SCD) substrates has favoured the improvement of chemical

(a) E-mail: giuseppe.prestopino@uniroma2 .it vapour deposition (CVD) techniques for homoepitaxial diamond growth and the spread of higher-performance SCD-based photodetectors. Several device geometries based on metal-semiconductor-metal (MSM) photoconductive and Schottky photodiode structures are reported [3-6] but little work has been preformed on SCD-based imaging systems although standard photolithography techniques and metallization processes allow to design high-resolution diamond-based multipixel detectors. Recently, SCD multilayered p-type diamond/intrinsic diamond/rectifying metal (PIM) Schottky photodiodes (SPDs) have been proposed by our group showing fast response times, excellent radiation hardness and good sensitivity to both SXR and DUV radiation $[7-9]$.

In this letter we report on the fabrication and characterization under synchrotron X-ray (XR) photons of a high-performance 1D imaging array, operated at zero bias voltage, based on a CVD single-crystal-diamond PIM photodiode with a multistrip (MS) Schottky metal pattern on top surface (MS-SPD in the following). The high-resolution capabilities of the tested device are demonstrated, limited only by photolithographic strip dimension and lateral drift-diffusion of photogenerated carriers. 


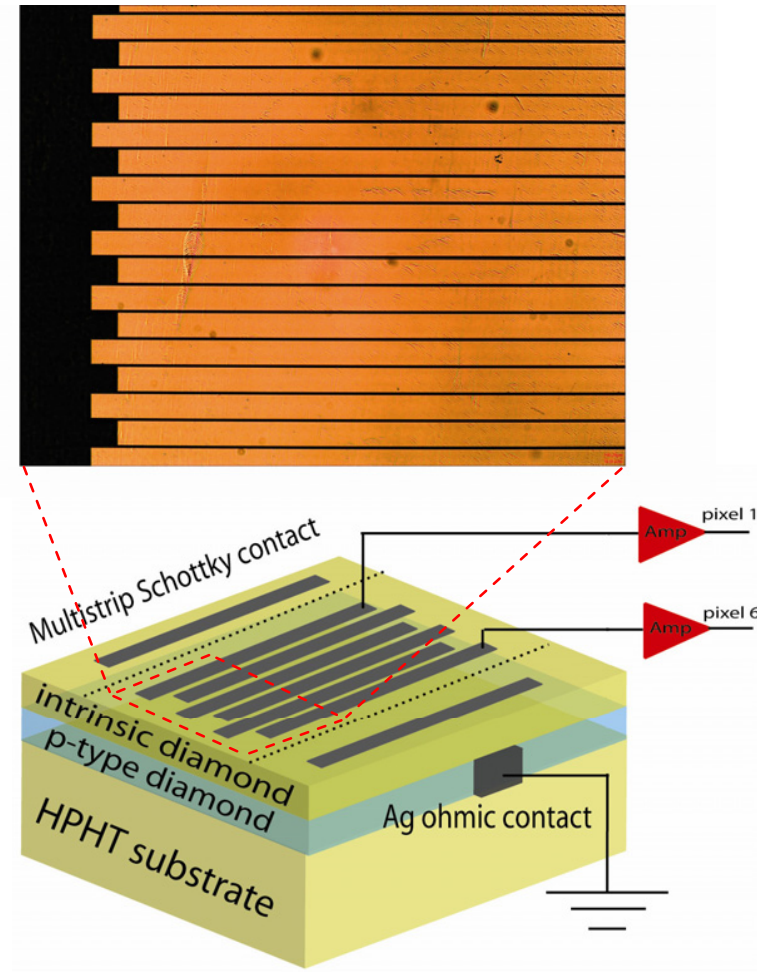

Fig. 1: (Colour on-line) Schematic representation of the multilayered synthetic single-crystal-diamond 1D multistrip Schottky photodiode (MS-SPD) and photo of the metal strips.

A two-step micro-wave plasma-enhanced CVD process was used to realize the homoepitaxial $p$-type/intrinsic diamond epilayers. A low-cost (100) synthetic HPHT-type IB single-crystal diamond, $4 \times 4 \times 0.45 \mathrm{~mm}^{3}$ in size, was used as substrate for the growth of a $25 \mu \mathrm{m}$ thick highly conductive boron-doped SCD layer with an acceptor density $\left(N_{\mathrm{A}}\right)$ of about $5 \times 10^{19} \mathrm{~cm}^{-3}$. A nominally intrinsic $40 \mu \mathrm{m}$ thick SCD film was then deposited in a separated reactor on the $p$-doped one, with a net electrically active acceptor-like defect concentration of about $10^{14} \mathrm{~cm}^{-3}[9]$. Ohmic silver contacts were formed on the highly-borondoped layer by an isothermal annealing at $500^{\circ} \mathrm{C}$ for $1 \mathrm{~h}$ in air. This treatment also allows the oxidation of the diamond surface. Finally, 32 thermally evaporated $\mathrm{Cr} / \mathrm{Al}$ metal strips (in sequence, $20 \mathrm{~nm} \mathrm{Cr}$ and $50 \mathrm{~nm}$ $\mathrm{Al}$ ), $90 \mu \mathrm{m}$ wide and $3 \mathrm{~mm}$ long, were patterned on the oxidized intrinsic diamond surface by a standard lift-off photolitographic technique with an interelectrode distance of $10 \mu \mathrm{m}$. A scheme of the device structure and a photo of the metal strips are reported in fig. 1. The coplanar array of Schottky electrodes defines the pixel sequence of the MS-SPD and the boron-doped SCD acts as grounded common buffer layer.

As illustrated in fig. 1, six adjacent pixels in the central zone of the array were measured. This choice was due to best perform a complete characterization of the device within the duration of the experimental campaign. A basic electrical current-voltage $(I-V)$ characterization was

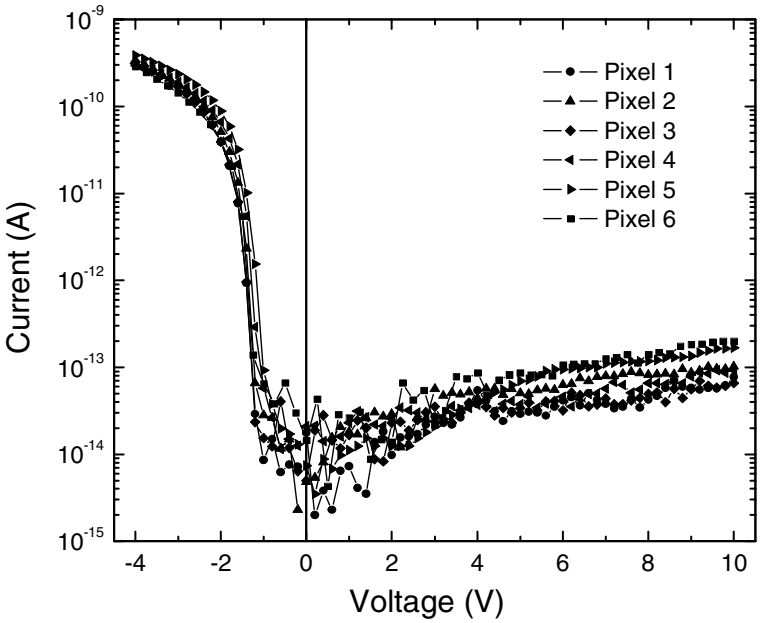

Fig. 2: Current-voltage characteristics of six pixels of the MS-SPD measured at room temperature.

performed at room temperature prior to X-ray irradiation with a Keithley $6517 \mathrm{~A}$ voltage source/picoammeter, following the procedures previously described for a singledotted PIM SPD [9].

The photoresponse properties of the tested pixels were evaluated using monochromatic XRs from the B16 beamline of Diamond Light Source (DLS) synchrotron. Two focus modes were considered. In the first one the monochromatic beam was focused by a toroidal mirror that enabled getting a focal spot of about $192 \mu \mathrm{m}$ (FWHM) in diameter (coarse-focus mode in the following). In this configuration the energy of the XR beam could be changed as the mirror focal length did not depend on the photon wavelength. The XR energy was varied from 6 to $20 \mathrm{keV}$. A compound refractive lens was then installed to get a microfocused beam with 3.2 and $2.6 \mu \mathrm{m}$ FWHM in the horizontal and vertical direction, respectively (micro-focus mode in the following). The size of the micro-focused beam was measured by taking transmission scans of $200 \mu \mathrm{m}$ diameter $\mathrm{Au}$ cross-wires and calculated by deriving the wirescans. In this case, the beam energy was fixed at $10 \mathrm{keV}$ and a high-resolution map of the MS-SPD response was measured. During all tests, the MS-SPD was placed in an earthed metal box on a high-precision translational stage and illuminated from the front patterned side at normal incidence. The measured pixels were operated in current mode and connected to low-noise variable-gain Keithley 428 transimpedance amplifiers. A calibrated silicon detector and a gas ionization chamber (IC) were used to calibrate XR power and monitor the incident photon flux.

Figure 2 shows the dark $I-V$ characteristics of the six MS-SPD tested pixels. Almost identical curves were obtained, revealing a very good uniformity of the Schottky metal junction along the oxidized intrinsic diamond surface. A clearly rectifying behaviour was observed with a rectification ratio of $7 \times 10^{3}$ at $\pm 4 \mathrm{~V}$ with a pixel-to-pixel variation of $2 \times 10^{3}$. Extremely low leakage currents below 


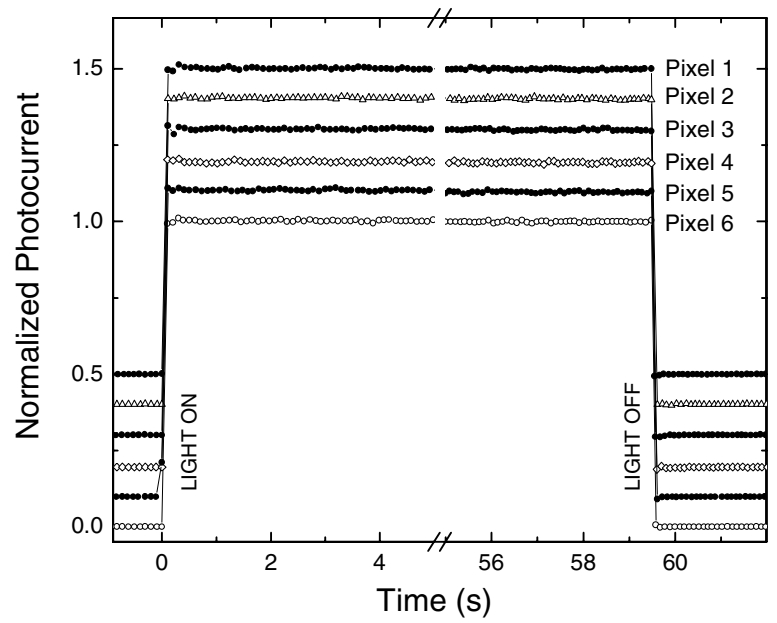

Fig. 3: Time response of the tested pixels to a $8 \mathrm{keV}$ XR photon beam at irradiation times of $\sim 60 \mathrm{~s}$.

$30 \mathrm{fA}$ were found at the operating bias voltage of $0 \mathrm{~V}$, slightly increasing at higher reverse biases (of the order of $100 \mathrm{fA}$ at $10 \mathrm{~V})$. In the forward regime, currents rapidly increase due to hole injection from the boron-doped SCD, following thermionic emission (TE) at low forward biases and a Ohmic-type behaviour at higher voltages. The TE currents were fitted by the conventional thermionic emission theory [10]. The Schottky barrier height and the ideality factor of the tested SPDs were evaluated to be $1.72 \pm 0.06 \mathrm{eV}$ and $1.9 \pm 0.1$, respectively.

The response time of the individual pixels was measured upon $8 \mathrm{keV}$ unfocused XR irradiation. In fig. 3 all the measured photocurrents are reported, normalized to their respective mean value and shifted by 0.1 in the $y$-direction for clarity. All the measurements were performed with a zero-bias voltage applied. Very reproducible results were obtained both in response time and signal stability. Identical rise and fall time were observed for all pixels, completely ascribed to the measurement system time constant.

The spectral response of the MS-SPD is displayed in fig. 4, where the responsivities $(\mathrm{A} / \mathrm{W})$ of the six pixels are reported as a function of the XR energy in the range 6-20 keV. The beam was operated in coarse-focus mode and each measured strip was in turn aligned to the beam centre by moving the device with the translational stage. The absolute device responsivity vs. the XR energy was obtained by comparing the currents measured by the MSSPD and the calibrated Si, exposed to the same photon flux, applying the proper calibration factors and the ratio of optical areas. The beamline crystal was appropriately detuned during the calibration of the detector to minimize the harmonic content. A high repeatability in spectral responsivity can be observed, with an average pixel-topixel variation of $0.6 \%$ in the whole investigated energy range. The measured responsivity values were analyzed in the framework of a simplified model that was developed

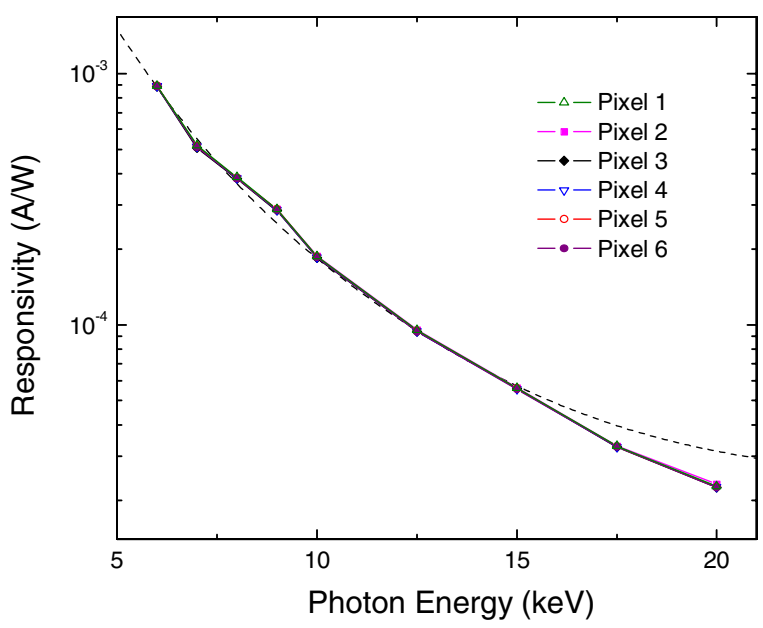

Fig. 4: (Colour on-line) Spectral responsivity of the measured pixels under irradiation of the XR beam in the $6-20 \mathrm{keV}$ energy range and fit of the experimental data calculated using eq. (1).

for Schottky barrier solar cells [11,12], taking into account absorption losses in the Schottky metal electrode and charge collection processes of photogenerated carriers. The dashed line plotted in fig. 4 is the fit of the experimental data calculated according to

$$
s(h \nu)=\frac{e}{w} T_{m}\left(1-\frac{e^{-\alpha_{\mathrm{SCD}} d_{d}}}{1+\alpha_{\mathrm{SCD}} L}\right),
$$

where $e$ is the elementary charge, $w$ the mean electronhole pair production energy, $T_{m}=e^{-\alpha_{\mathrm{Al}} d_{\mathrm{Al}}} e^{-\alpha_{\mathrm{Cr}} d_{\mathrm{Cr}}}$ the $\mathrm{X}$-ray transmission of the top metal electrode $\left(d_{\mathrm{Al}}=\right.$ $\left.50 \mathrm{~nm}, d_{\mathrm{Cr}}=20 \mathrm{~nm}\right)$ and $\alpha_{\mathrm{Al}}, \alpha_{\mathrm{Cr}}, \alpha_{\mathrm{SCD}}$ the energydependent absorption coefficients of $\mathrm{Al}, \mathrm{Cr}$ and diamond, respectively. The terms $d_{d}$, the depletion layer thickness in diamond beneath the Schottky junction, and $L$, the bulk diffusion length, define the SCD active thickness $d_{\mathrm{SCD}}=$ $d_{d}+L$. Known X-ray optical data for the elements [13], a value of $13.2 \mathrm{eV}$ for $w / e$ [14] and a diffusion length $L$ of $2.6 \mu \mathrm{m}[9]$ were used. Only the $d_{d}$ fit parameter was left free and a value of $0.51 \pm 0.09 \mu \mathrm{m}$ was found, obtaining a total active diamond thickness $d_{\mathrm{SCD}}$ of about $3.1 \mu \mathrm{m}$. Finally, it should be noted that due to photon and electron escape losses, not considered in the simplified model given by eq. (1), the fitting curve was not able to reproduce the higher-energy points above $15 \mathrm{eV}[9]$.

The resolution properties of the MS-SPD were studied by mapping the device response with a raster scan of the diamond surface. Currents from four pixels were separately measured during an overnight exposure connecting each of them to Keithley 428 transimpedance amplifiers. The XR beam was operated in micro-focused mode at an energy of $10 \mathrm{keV}$. The scan $x$-direction ( $50 \mu$ m wide steps) corresponded to the strip length, whereas the $y$ one $(2 \mu \mathrm{m}$ wide steps) to the strip width. A 3D map and the relative contour plot of the measured currents is displayed in fig. 5 . No overlapping among pixel currents and a high discrimination between adjacent strips were observed. Moreover, 


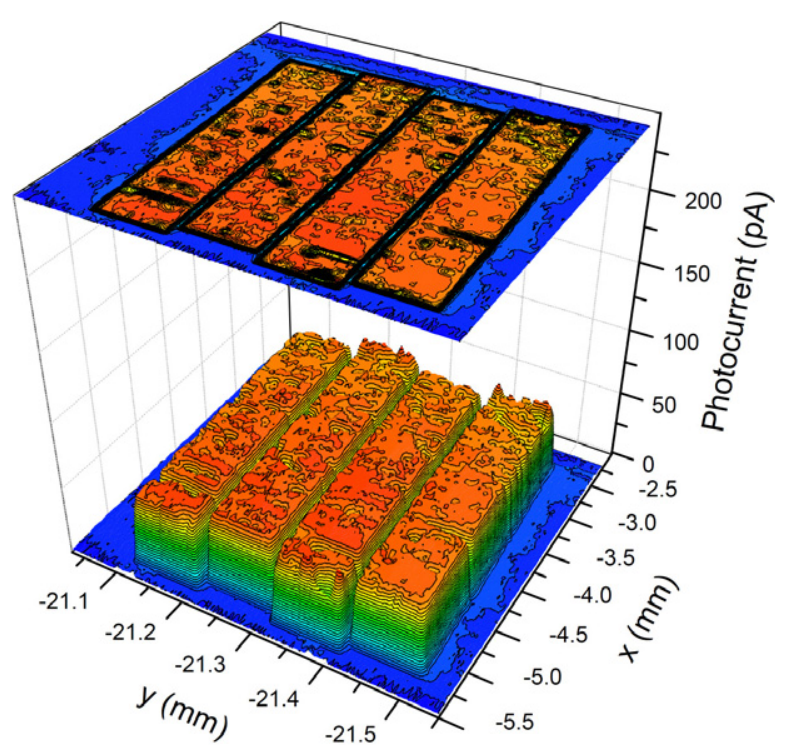

Fig. 5: (Colour on-line) 3D map and contour plot of the MS-SPD response measured by a raster scan of four pixels with the XR beam operated in micro-focus mode.

a $1 \%$ variation in the mean value of pixel currents and a good signal uniformity with $6 \%$ average signal fluctuation along each strip were found. The small non-uniformities observable in the sensitivity map reported in fig. 5 could be ascribed to defects or inhomogeneities related to the metal-diamond interface.

The profile of the current measured along the $y$-direction at the edge of one of the four pixels is displayed in fig. 6. From the full width at half maximum (FWHM) of the Gaussian fit of the derivative of the experimental data a strip broadening of $4.3 \mu \mathrm{m}$ was calculated, with a clear broadening with respect to the intrinsic vertical size of the micro-focused XR beam $(2.6 \mu \mathrm{m})$. This effect is due both to the lateral diffusion of minority photogenerated carriers towards the depleted region and to the non-uniformity of the electric-field distribution at the edge of the metal strip, as previously observed in ion-beam-induced charge (IBIC) maps on similar multi-electrode structures [15]. The smoothing of pixel sensitivity from the plateau behaviour does not occur entirely outside the metal strip but also partially extends underneath the electrode, as clearly indicated by the position of the electrode edge reported in fig. 6 (vertical dotted line).

In summary, a multipixel synthetic single-crystaldiamond Schottky photodiode, operating in unbiased mode, was fabricated and characterized at the Diamond Light Source synchrotron by using high-flux monochromatic X-ray beams from 6 to $20 \mathrm{keV}$ and a micro-focused $10 \mathrm{keV}$ beam. The detector showed low dark current, fast response time and signal stability. A high repeatability in spectral responsivity was observed, with an average pixel-to-pixel variation of $0.6 \%$ in the whole investigated

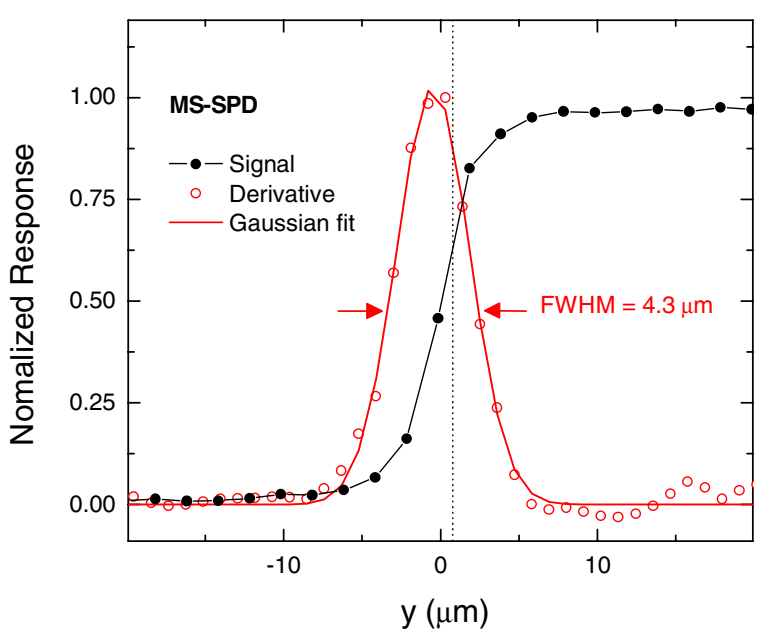

Fig. 6: (Colour on-line) Profile of the current measured at the edge of one single strip along the scan $y$-direction. The derivative of the experimental data and its Gaussian fit are reported too.

energy range. Finally, a good signal uniformity with $6 \%$ average signal fluctuation along each strip and $4.3 \mu \mathrm{m}$ broadening at the edge of the strip when irradiated with a $2.6 \mu \mathrm{m}$ spot were found, suggesting an excellent position sensitivity of the tested device structure.

$$
* * *
$$

The authors wish to thank M. Benetti, D. CAnnatì and F. Di Pietrantonio of the Institute of Acoustics and Sensors "Orso Mario Corbino" (IDASC) of the National Research Council (CNR) for providing photolithography techniques, the Diamond Light Source for providing beam time at the beamline B16 and Mr. A. Malandine for his technical support during the experiment.

\section{REFERENCES}

[1] Kania D. R., in The Physics of Diamond, Proceedings of the International School of Physics "Enrico Fermi", Course CXXXV, edited by Paoletti A. and TucciaRONE A. (IOS Press, Amsterdam; SIF, Bologna) 1997, p. 565.

[2] Bergonzo P. and Jackman R. B., in Thin-Film Diamond II, Semiconductors and Semimetals, edited by Nebel C. E. and Ristein J., Vol. 77 (Elsevier, Amsterdam) 2004, p. 197.

[3] Bevilacqua M. and Jackman R. B., Appl. Phys. Lett., 95 (2009) 243501.

[4] Teraji T., Yoshizaki S., Wada H., Hamada M. and Iто T., Diamond Relat. Mater., 13 (2004) 858.

[5] Liao M. and Koide Y., Appl. Phys. Lett., 89 (2006) 113509.

[6] KoIde Y., Appl. Surf. Sci., 254 (2008) 6268.

[7] Tartoni N., Angelone M., Pillon M., Almaviva S., Marinelli M., Milani E., Prestopino G., Verona C., 
Verona-Rinati G. and Roberts M. A., IEEE Trans. Nucl. Sci., 56 (2009) 849.

[8] Almaviva S., Marinelli M., Milani E., Prestopino G., Tucciarone A., Verona C., Verona-Rinati G., Angelone M., Pillon M., Dolbnya I., Sawhney K. and Tartoni N., J. Appl. Phys., 107 (2010) 014511.

[9] Angelone M., Pillon M., Marinelli M., Milani E., Prestopino G., Verona C., Verona-Rinati G., Coffey I., Murari A. and Tartoni N., Nucl. Instrum. Methods A, 623 (2010) 726.

[10] Sze S. M. and NG K. K., Physics of Semiconductor Devices, 3rd edition (Wiley, New York) 2007.
[11] Hovel H. J., Semiconductors and Semimetals, Vol. 11 (Academic Press, New York) 1975, pp. 112-126.

[12] Krumrey M. and Tegeler E., Rev. Sci. Instrum., 63 (1992) 797.

[13] Henke B. L., Gullikson E. M. and Davis J. C., CXRO database for X-ray attenuation length of a solid, http://henke.lbl.gov/optical_constants/atten2.html.

[14] Kozlov S. F., Stuck R., Hage-Ali M. and Siffert P., IEEE Trans. Nucl. Sci., 22 (1975) 160.

[15] Vittone E., Skukan N., Pastuović Ž., Olivero P. and JAKŠIĆ M, Nucl. Instrum. Methods B, 267 (2009) 2197. 